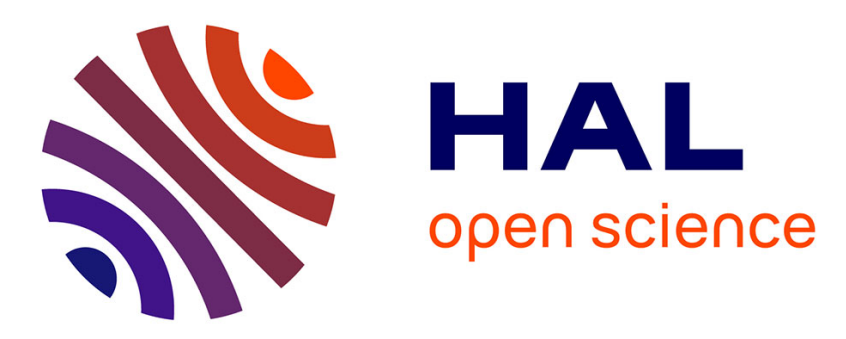

\title{
Double-negative pillared elastic metamaterial
}

Wei Wang, Bernard Bonello, Bahram Djafari-Rouhani, Yan Pennec, Jinfeng

Zhao

\section{To cite this version:}

Wei Wang, Bernard Bonello, Bahram Djafari-Rouhani, Yan Pennec, Jinfeng Zhao. Double-negative pillared elastic metamaterial. Physical Review Applied, 2018, 10 (6), pp.064011. 10.1103/PhysRevApplied.10.064011 . hal-02053395

\section{HAL Id: hal-02053395 \\ https://hal.sorbonne-universite.fr/hal-02053395}

Submitted on 1 Mar 2019

HAL is a multi-disciplinary open access archive for the deposit and dissemination of scientific research documents, whether they are published or not. The documents may come from teaching and research institutions in France or abroad, or from public or private research centers.
L'archive ouverte pluridisciplinaire HAL, est destinée au dépôt et à la diffusion de documents scientifiques de niveau recherche, publiés ou non, émanant des établissements d'enseignement et de recherche français ou étrangers, des laboratoires publics ou privés. 


\title{
Double-negative Pillared Elastic Metamaterial
}

\author{
Wei Wang ${ }^{1}$, Bernard Bonello ${ }^{1 *}$, Bahram Djafari-Rouhani ${ }^{2}$, Yan Pennec ${ }^{2}$, and Jinfeng Zhao ${ }^{3}$ \\ ${ }^{1}$ Sorbonne Université, UPMC Université Paris 06 (INSP-UMR CNRS 7588), \\ 4, place Jussieu 75005 Paris, France \\ ${ }^{2}$ Institut d'Electronique, de Micro-électronique et de Nanotechnologie (IEMN-UMR CNRS 8520), \\ Université de Lille Sciences et Technologies, Cité Scientifique, 59652 Villeneuve d'Ascq Cedex, France \\ ${ }^{3}$ School of Aerospace Engineering and Applied Mechanics, Tongji University, 100 Zhangwu Road, \\ 200092 Shanghai, China
}

*corresponding author: bernard.bonello@insp.jussieu.fr

\begin{abstract}
We present a single-phase double-sided pillared metamaterial with effective mass density and elastic modulus simultaneously negative. The negative effective mass density is achieved owing to the combination of the symmetric bending resonance and the antisymmetric compressional resonance of both pillars, whereas the negative effective elastic modulus results from their symmetric compressional resonance. Comparisons with the single-sided pillared structure are made. The dependence of the width of the double-negative band against the geometric parameters of the unit cell is also investigated. In addition, the negative refraction and the zero-index refraction of the symmetric Lamb wave in the deepsubwavelength scale are observed, while at the same frequency, the propagation of the antisymmetric Lamb wave is forbidden.
\end{abstract}

\section{INTRODUCTION}

Elastic metamaterials are artificial composites featuring frequency dependent constitutive properties that are not present in nature. Owing to their locally resonant substructures, their effective parameters, including the mass density, the bulk, and the shear modulus, can be dynamically set to a positive, zero or negative value [1-4], enabling thus to manipulate the propagation of elastic waves in the subwavelength scale. In analogy to their electromagnetic counterparts, elastic metamaterials with simultaneously negative effective mass density (NMD) and elastic modulus (NEM) have attracted considerable attention notably because of their great potential for the negative refraction of elastic waves or the over-the-diffraction-limit imaging [5-10]. The double negativity can be realized either by combining two different substructures, each supporting a different resonant mode or by constructing a single structure where two resonances occur at a single frequency[11,12]. In the wake of the seminal work by Liu et al. [13], a three-constituents chiral metamaterial made of soft-coated heavy cylinders embedded into a polyethylene matrix that 
Phys. Rev. Appl. NT10126 - Revised version

exhibits the double negativity thanks to the combination of the translational and the rotational resonances of the heavy core has been proposed [14]. Using the same two resonances but this time in a single-phase metamaterial, Zhu et al.[8] observed experimentally the subwavelength negative refraction of longitudinal elastic waves and demonstrated in this way the occurrence of simultaneous NMD and NEM in this system. A single-phase solid metamaterial which unit cell comprises two independent resonators, each of them supporting a distinct resonant mode, was proposed by Oh et al.[15] to independently tune the effective mass density and stiffness. However, in most of these aforementioned systems, the double negativity only occurs in a narrow frequency band which may constitute a drawback for many applications. To overcome this difficulty, Dong et al. [9] developed a topology optimization scheme to design a two-dimensional (2D) single-phase anisotropic elastic metamaterial with broadband doubly negative effective property. They further demonstrated numerically the superlensing effect in the deep-subwavelength regime as well as the cloaking effect for both the longitudinal and transverse waves.

Beside these designs that mainly aim at manipulating bulk waves, doubly negative elastic metamaterials involving other types of elastic waves, such as Lamb waves, have attracted since a few years more and more interest. Actually, a decade ago a new type of phononic crystal consisting of an array of pillars on a plate was simultaneously proposed by Pennec et al.[16] and Wu et al.[1]. These structures have the ability of exhibiting both Bragg gaps and hybridization gaps associated to local resonances of the pillars, where the latter can be pushed into low frequency (subwavelength) range. These structures can therefore serve both as phononic crystals or metamaterials and provide a useful and practical platform for several physical properties such as waveguiding, filtering, acoustic lensing or liquid sensing. A few years ago, Gusev et al.[17] introduced a doubly negative metamaterial for flexural waves based on resonant elements attached to a plate. In this system, negative density is achieved by normal-force interactions, whereas negative modulus is obtained thanks to the introduction of rotationally resonant mechanical elements. More recently, a class of 2D single-phase metamaterials have shown noteworthy dispersion properties including a Dirac-like cone at the center of the Brillouin zone (BZ), zero-group velocity in the fundamental Lamb modes [18], and anomalous refraction on transmitted antisymmetric Lamb mode involving mode conversion of a symmetric mode [19]. Among the most promising dynamic systems for applications requiring the full control over the phase are the zero-index elastic/acoustic metamaterials [20]. Actually, the wave transmitted through a medium exhibiting this dynamic property remains unaffected since it is not subjected to any phase change. As a consequence of this remarkable property, these structures could serve as a basis to shape an "inaudibility cloak". Let us also mention a few other relevant works using the platform of phononic plates. For instance, Assouar et al.[21] reported in a theoretical work the enlargement of the locally resonant band gap in 2D sonic crystals based on a double-sided stubbed plate. The relative bandwidth was significantly increased by a factor of two compared to the classical one-sided 
Phys. Rev. Appl. NT10126 - Revised version

stubbed plate. The elastic energy carried by SAW can also be localized and confined within micropillars, as it has been demonstrated lately by Benchabane et al.[22]. Bilal et al.[23] proposed a metamaterial in which the pillars stand in between the holes regularly patterned in a plate. This structure, sometimes referred as a trampoline, allows to broaden the subwavelength band gap by a factor of four. Furthermore, it has been shown that such pillared metamaterials exhibit NMD at low frequencies where homogenization theories apply [24,25]. It is noteworthy that, owing to the coupling between the Lamb waves and the resonant modes, a strong anisotropy of the effective mass density may be observed around the resonant frequencies. This makes the pillared structures suitable for the manipulation and the subwavelength focusing of elastic wave as well as excellent candidates for numerous applications ranging from biomedical imaging to energy harvesting [26,27].

In this work, we have investigated a single-phase double-sided pillared metamaterial. We show that this structure exhibits both NMD and NEM allowing for the propagation of the zero-order symmetric Lamb wave in a frequency interval where the propagation of the antisymmetric Lamb wave keeps forbidden. For comparison, we firstly have investigated a single-sided pillared metamaterial featuring NMD. The mechanism responsible for this single negativity, and in turn for the formation of the low frequency band gap, is described as being the combination of a bending mode and a compressional mode of the pillar. We then have investigated a double-sided pillared metamaterial that displays the double negativity. On the basis of the preceding analysis, it is shown that NMD is achieved within a frequency range where occur both the symmetric bending resonance and the antisymmetric compressional resonance of the pillars, whereas NEM only involves the symmetric compressional resonance. We develop a simplified massspring model to theoretically describe this behavior. Additionally, the double negativity of the metamaterial is further established through both numerical simulations of the negative refraction of symmetric Lamb mode and the zero-index refraction in the deep-subwavelength regime. Finally, the width of frequency range where the metamaterial is doubly negative against the geometric parameters of the unit cell is presented.

\section{SINGLE-SIDED PILLARED METAMATERIAL}

\section{A. Lamb waves in a periodic structure}

Before analyzing the band structure and the dynamical properties of a pillared metamaterial, we briefly recall in this section the main features of Lamb waves in periodically structured plates [28].

Lamb waves are the solutions of the equations of motion for a harmonic wave propagating in a medium with a finite thickness along one direction of space and infinite along the other two, with the boundary conditions that the components of the stress in the direction normal to the free surfaces are zero. Owing to the symmetry with respect to the mid-plane of the plate, these modes can be distinguished as symmetric (S) 
Phys. Rev. Appl. NT10126 - Revised version

and antisymmetric (A) modes. In a homogeneous isotropic medium, the three lowest branches are respectively the antisymmetric flexural branch and the symmetric shear horizontal and longitudinal branches. The higher branches have a cut-off frequency as the wave vector $\mathrm{k}$ approaches 0 . All these modes are highly dispersive [28]. The phononic plate is created by inserting periodic inclusions (such as holes) in the plate or by depositing pillars on it. The dispersion curves of this periodic inhomogeneous medium are now described in the corresponding 2D BZ with their dynamical properties having specific characteristics including band gaps, the folding of the dispersion curves and the mode conversion. In particular, the strong resonances associated with pillars can be very efficient in opening band gaps. Although the branches have more complex character and coupling between the displacement components than in a homogeneous plate, some branches still keep a significant flexural, longitudinal or shear horizontal character. In the case of one-sided pillars, the symmetry with respect to the mid-plane of the plane is strictly speaking broken, while this symmetry property can be restored in the case of double-sided pillars. This allows to study separately the symmetric and antisymmetric modes and in particular the design of their corresponding band gaps. We shall see that this property will be very helpful in the design of the double negative material which is the objective of this paper.

\section{B. Band structure and localized modes}

We analyze in this section the dynamical properties of a metamaterial made of a square lattice with pillars erected on one face of a thin homogeneous plate. The elementary unit cell is displayed in Fig. 1(a) where $a$ denotes the lattice constant, $h$ and $d$ stand for the height and the diameter of the pillar respectively, and $e$ is the thickness of the matrix plate. The periodicity is in the plane $(x, y)$ and $z$-axis is chosen perpendicular to the plate. In order to obtain dynamic properties in the MHz range, we assumed these values (in $\mu \mathrm{m}$ ) $a=$ $200, h=325, d=120$, and $e=100$. The complete structure was made of isotropic steel whose physical parameters are the elastic modulus $E=200 \mathrm{GPa}$, the Poisson's ratio $v=0.3$, and the mass density $\rho=$ $7850 \mathrm{~kg} / \mathrm{m}^{3}$. Both the plate and the pillars being made from the same material, a strong coupling between the Lamb waves propagating in the plate and the vibrations of the pillars may be expected close to the resonant frequencies [12]. This configuration much differs from composite heterostructures where a soft rubber provides the bonding between the resonators and the stiff plate [29-31] generally giving rise to sharp resonances.

The band structure shown in Fig. 1(b) was obtained by applying periodic conditions in both $x$ - and $y$ directions and solving the eigenfrequencies equation with the help of a finite element method (FEM). The color scale in this figure represents the relative amplitude of the out-of-plane component 
Phys. Rev. Appl. NT10126 - Revised version

$\xi=\frac{\iiint_{\text {pillar }} u_{z}^{2} d v}{\iiint_{\text {pillar }}\left(u_{x}^{2}+u_{y}^{2}+u_{z}^{2}\right) d v}$. A complete band gap opens up in between $3.17 \mathrm{MHz}$ to $3.48 \mathrm{MHz}$. In this frequency range, the wavelengths of the lowest order antisymmetric $\left(\mathrm{A}_{0}\right)$ and symmetric $\left(\mathrm{S}_{0}\right)$ Lamb modes in the matrix plate are about $500 \mu \mathrm{m}$ and $1800 \mu \mathrm{m}$ respectively i.e. more than twice and nine times the lattice constant $a$. Therefore, this gap cannot result from Bragg interferences. This rather suggests that this complete band gap should be ascribed to local resonances of the pillars. To support this assertion, we have computed the displacement field distribution and the deformation of the unit cell at point $M$ of the BZ for the three branches converging to $f \sim 3.1 \mathrm{MHz}$ (see inset in Fig. 1(b)). The result is displayed in Fig. 1(c). Clearly, the deformation concentrates in the pillar whereas it is almost zero in the plate. This is further supported by the flat branches in the low frequency edge of the gap that correspond to zero group velocities and are evidences of local resonances as widely documented in literature [21,32-34]. Furthermore, the deformations shown in Fig. 1(c) allow to identify two second-order bending modes, labelled as A and B in Fig. 1(c), and one compressional mode, labelled as C. Given the square symmetry of the unit cell, modes A and B are polarized along orthogonal directions and occur at the same frequency. 
(a)

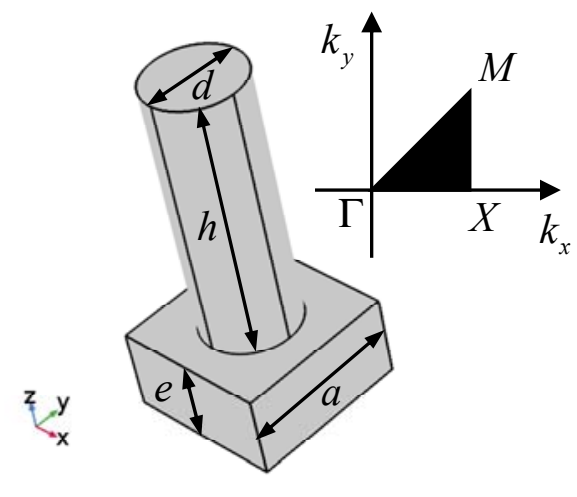

(b)

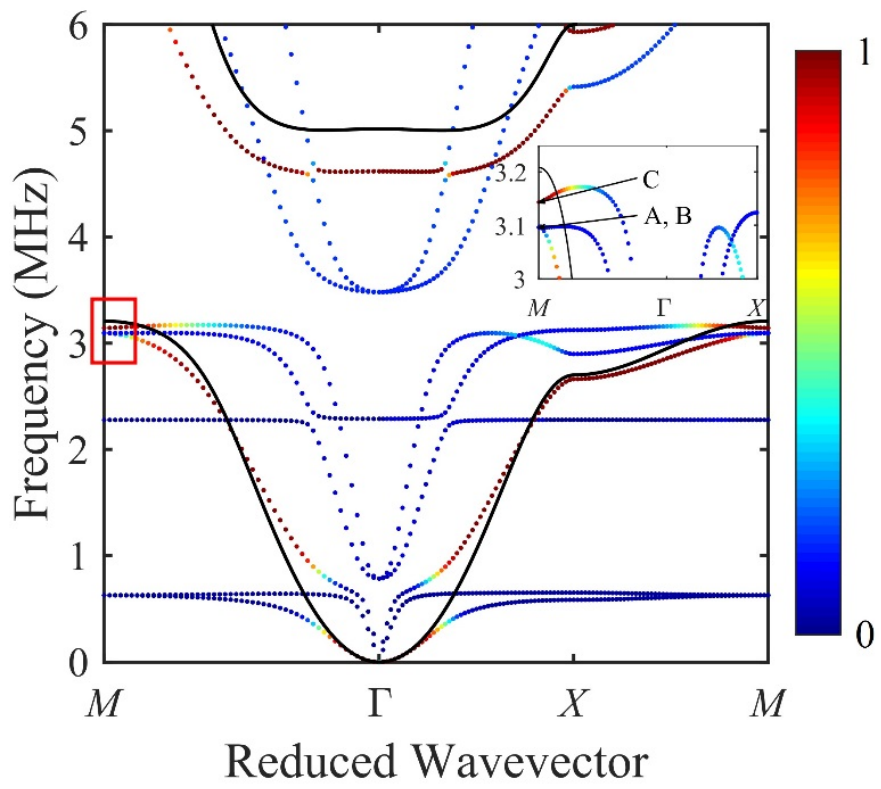

(c)
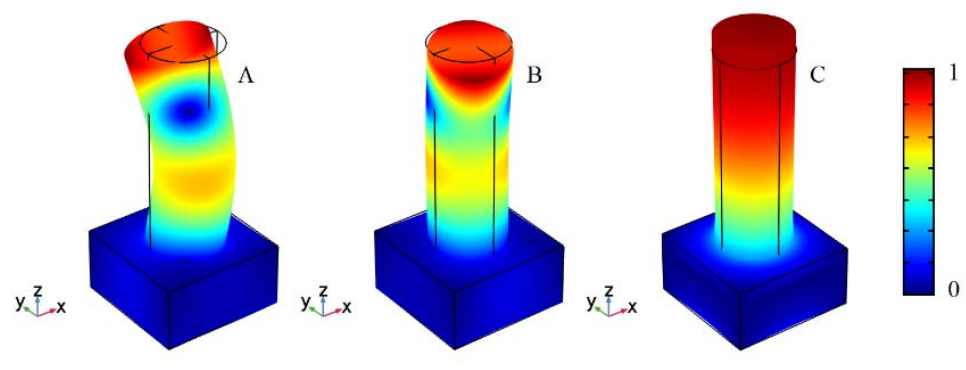

Figure 1: (a) Unit cell of the single-sided pillared metamaterial and first irreducible BZ of the square lattice. (b) Band structure in the low frequency range along the high symmetry axes of the BZ; the color scale corresponds to the relative amplitude of the out-of-plane component, integrated over its volume (see text). For comparison, the band structure of the simplified coupled system described by Eq. (1) is shown as black solid lines. Inset: enlargement of the band gap around 3.1MHz. (c) Normalized total displacement field amplitude and deformation in the unit cell for modes denoted as $\mathrm{A}, \mathrm{B}$, and $\mathrm{C}$ in the band structure.

\section{Formation of the low frequency complete band gap}

We investigate here the formation of the low frequency band gap using FEM with frequency response analysis. The method, which is only valid within the long wavelengths limit, consists of applying an external displacement field $\mathbf{U}$ on the four lateral boundaries of the unit cell while leaving free both the face supporting the pillar and the rear face of the plate $[4,24,35]$. The induced force $\mathbf{F}$ exerted on these boundaries is then derived from the stress average over the four boundaries of the unit cell considered as a homogeneous media. In the harmonic regime at frequency $\omega / 2 \pi, \mathbf{F}$ and $\mathbf{U}$ are related through $\mathbf{F}=-\omega^{2} V[\rho]_{\text {eff }} \mathbf{U}$, where $V$ is the volume of the unit cell and $[\rho]_{e f f}$ the $3 \times 3$ dynamic effective mass density matrix to be determined. This can be done by successively applying the harmonic displacement 
Phys. Rev. Appl. NT10126 - Revised version

$\mathbf{U}=U_{0} \exp (i \omega t) \mathbf{x}_{i}(i=1,2,3)$ along one of the axes in the principal coordinate system while the other two components are set to zero. In addition, symmetry arguments allow to reduce the number of independent matrix elements. For the structure we consider here, $z$-axis being a four-fold symmetry axis the matrix elements are invariant through the exchange of indices 1 and 2 and therefore $\rho_{11}=\rho_{22}, \rho_{12}=\rho_{21}$, $\rho_{13}=\rho_{23}$ and $\rho_{31}=\rho_{32}$. Moreover, it has been shown in Ref. [21] that in a pillared metamaterial the offdiagonal terms in $[\rho]_{e f f}$ are null except for values very close to the resonant frequencies where a singularity occurs; therefore the only elements to consider are $\rho_{11}, \rho_{22}=\rho_{11}$ and $\rho_{33}$. Their variations around the lower edge of the band gap are displayed in Fig. 2. The component $\rho_{11}$ that mainly relates to in-plane motions (modes A and B in Fig. $1(\mathrm{c})$ ), is negative from $3.21 \mathrm{MHz}$ to $3.50 \mathrm{MHz}$, whereas $\rho_{33}$ that should be ascribed to out-of-plane displacements (mode C in Fig. 1(c)), becomes negative from 3.26MHz to $4.63 \mathrm{MHz}$. A complete band gap opens when all the components of the effective mass density matrix turn negative, i.e. in between $3.26 \mathrm{MHz}$ and $3.50 \mathrm{MHz}$ in the present case.

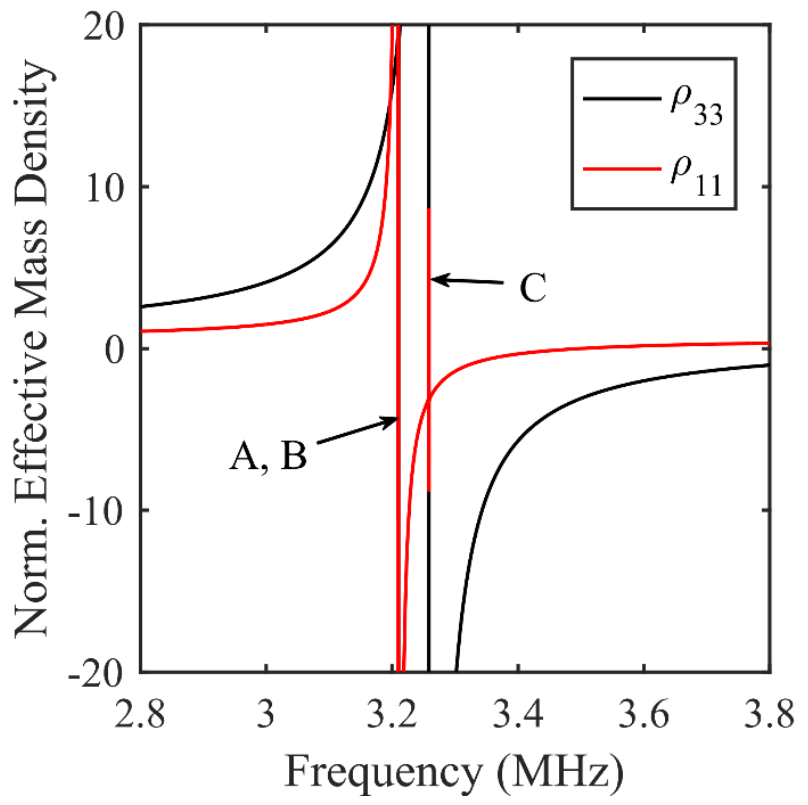

Figure 2: Components $\rho_{11}$ and $\rho_{33}$ of the effective mass density normalized to the mass density of steel, as a function of the frequency.

This is in good agreement with the band gap shown in Fig. 1(b) that goes from 3.17MHz to 3.48MHz. The small discrepancy of about $2.8 \%$ on the frequency at the lower edge of the gap may be readily ascribed to the phase change across the unit cell, not accounted for in the calculations since the preceding theory is only valid within the long wavelengths limit.

Beside this comprehensive approach where the structure is considered as an effective medium, one can also explain the formation of the low frequency band gap owing to a local analysis of the motions of the 
Phys. Rev. Appl. NT10126 - Revised version

pillars. In this framework, the resonator is regarded as a mass-spring system coupled to the plate where a wave at frequency $\omega / 2 \pi$ and wave vector $\mathbf{k}$ propagates. Let us first consider the first-order compressional resonance shown in Fig. 1(c) for which the pillar vibrates along the $z$-direction and exerts a vertical traction force on the matrix plate. This can be modeled as a mass-spring subsystem with mass $m$ and spring stiffness $k_{\mathrm{C}}$ depicted by the right panel in Fig. 3(a). Gusev et al. [17] already set the equations of motion of this corresponding mechanical system and obtained an analytical expression for the effective mass density component $\rho_{33}$. The governing equations of this simplified model in the frequency domain can be written as:

$$
\left\{\begin{array}{l}
D \frac{\partial^{4}}{\partial x^{4}} \hat{u}_{z}-\rho e \omega^{2} \hat{u}_{z}=n k_{\mathrm{C}}\left(\hat{u}-\hat{u}_{z}\right) \\
m \omega^{2} \hat{u}=k_{\mathrm{C}}\left(\hat{u}-\hat{u}_{z}\right)
\end{array} .\right.
$$

In Eq. (1), Fourier transformations of both the vertical displacement of the matrix plate $u_{z}$ and mass $u$ allow to eliminate the time dependence and lead to the variables $\hat{u}_{z}$ and $\hat{u}$ written in the frequency domain; $D=e^{3} E / 12\left(1-v^{2}\right)$ is the flexural rigidity of the matrix plate that depends on both the elastic modulus $E$ and the Poisson's ratio $v ; n$ is the number of mass-springs per unit area; $\omega_{\mathrm{C}}=\sqrt{k_{\mathrm{C}} / m}$ is the resonant frequency of the mass-spring subsystem. Remembering that $\hat{u}_{z}$ reads $\hat{u}_{z}=u_{0} \exp (i k x)$, we can derive the wave number $k$ by solving Eq. (1), namely:

$$
k^{2}=\omega\left(\frac{e}{D}\right)^{1 / 2}\left[\rho+\frac{n m}{e\left(1-\omega^{2} / \omega_{\mathrm{C}}^{2}\right)}\right]^{1 / 2} .
$$

Equation (2) shows that $k^{2}$ turns imaginary if $\omega$ takes a value in between $\omega_{\mathrm{C}}$ and $\left(n m / \rho e^{+1}\right)^{1 / 2} \omega_{\mathrm{C}}$, hence preventing the propagation of the elastic waves in the plate. Moreover, Eq. (2) allows to define the effective mass density as:

$$
\rho_{\text {eff }}(\omega)=\rho+\frac{n m}{e\left(1-\omega^{2} / \omega_{\mathrm{C}}^{2}\right)},
$$

which is negative when the excitation frequency is in the same interval. This explains the occurrence of the negative value of component $\rho_{33}$. This simplified model can be further extended to the case of a 2D phononic crystal by setting periodic conditions and implementing a plane wave expansion method to derive the dispersion curves [36]. The solution is represented as black solid lines in Fig. 1(b). 
Phys. Rev. Appl. NT10126 - Revised version

It is less intuitive to establish the governing equations that accurately describe the complex motions of both the pillar and the matrix plate for the second-order bending resonance. However, the problem can be simplified by equating the single-sided pillared unit cell to the classical cantilevered beam in which the rigid body is replaced by a deformable medium and where the oscillation of the pillar is excited by the elastic wave that propagates in the plate. In this simplified model depicted in Fig. 3(b), the overall motion is broken down into two motions that can be studied independently, namely the motion of the pillar modeled by an internal moment and the motion in the plate driven by a traction force parallel to the surface. This decoupling allows for a comprehensive analyze of the effective mass density and a better understanding of the occurrence of NMD in this type of structures.

Let us first consider the motion of the pillar in the sagittal plane depicted by the mid panel in Fig. 3(b). Since no traction force is considered, the motion reduces to a pendulum motion and can be well modeled by a mass-torsional spring with mass $m$, rotational inertia $J$ and torsional stiffness $k_{\mathrm{B}}$ rigidly attached to the plate. Then, the governing equations for waves propagation along $x$-direction in the plate-resonator coupled system can be written as [37]:

$$
\left\{\begin{array}{l}
D \frac{\partial^{4} \hat{u}_{z}}{\partial x^{4}}-\rho e \omega^{2} \hat{u}_{z}=n k_{\mathrm{B}}\left(\frac{\partial \hat{\theta}}{\partial x}-\frac{\partial^{2} \hat{u}_{z}}{\partial x^{2}}\right), \\
J \omega^{2} \hat{\theta}=k_{\mathrm{B}}\left(\hat{\theta}-\frac{\partial \hat{u}_{z}}{\partial x}\right)
\end{array}\right.
$$

where $\hat{u}_{z}$ and $\hat{\theta}$ are normal displacement of the matrix plate and rotation angle of torsional spring in frequency domain; $k_{\mathrm{B}}$ and $J$ determine the resonant frequency of the mass-torsional spring subsystem through $\omega_{\mathrm{B}}=\left(k_{\mathrm{B}} / J\right)^{1 / 2}$. By solving Eq. (4), we can derive the wave number $k$, namely:

$$
k^{2}=\frac{1}{2 D}\left\{\left[4 D e \omega^{2} \rho+\left(\frac{n J \omega_{\mathrm{B}}^{2}}{\omega_{\mathrm{B}}^{2} / \omega^{2}-1}\right)^{2}\right]^{1 / 2}-\frac{n J \omega_{\mathrm{B}}^{2}}{\omega_{\mathrm{B}}^{2} / \omega^{2}-1}\right\} .
$$

Equation (5) allows to define the effective mass density at frequency $\omega / 2 \pi$ as being:

$$
\rho_{e f f}(\omega)=\rho+\frac{n^{2} J^{2} \omega^{2}}{4 D e}\left(\frac{1}{1-\omega^{2} / \omega_{\mathrm{B}}^{2}}\right)^{2}
$$

Equation (6) shows that $\rho_{\text {eff }}$ is always positive whatever the frequency. Therefore, the resonance of the torsional spring cannot explain on its own that $\rho_{11}$ becomes negative in a specific frequency range, and in 
turn the occurrence of NMD. Therefore, it is essential to consider the traction force acting on the foot of the pillar to understand the occurrence of NMD evidenced by the simulations above.

This force excites the plate into an oscillation and the pillar can be regarded as an additional mass $\mathrm{M}$ attached to the unit cell by compression springs with stiffness $k_{\mathrm{H}}$. For waves propagating along $x$-direction, the problem reduces to the classic 1D mass-spring metamaterial depicted in the right panel in Fig. 3(b). One can show that the effective mass density can be written as [2]:

$$
\rho_{e f f}=m_{0}+\frac{M}{1-\left(\omega^{2} / \omega_{\mathrm{H}}^{2}\right)}
$$

where $m_{0}$ stands for the mass of the matrix in the unit cell and $\omega_{\mathrm{H}}=\left(k_{\mathrm{H}} /\left(M+m_{0}\right)\right)^{1 / 2}$ is the angular frequency of the assembly "unit cell+additional mass". Clearly, the effective mass density turns negative between $\omega_{\mathrm{H}}$ and $\omega_{\mathrm{H}} \sqrt{1+M / m_{0}}$. This simple model well explains the occurrence of negative values of $\rho_{11}$, as well it shows that the existence of a traction force is essential to understand the occurrence of NMD in the single-sided pillared metamaterials. We show in the next section that it is also essential to explain the negative properties of the double-sided pillared metamaterials as well.

(a)
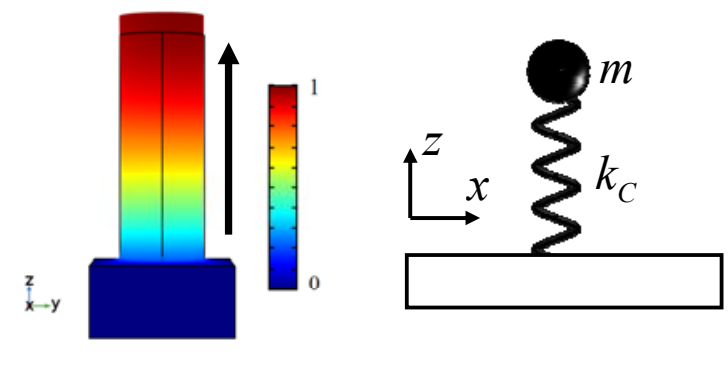

(b)
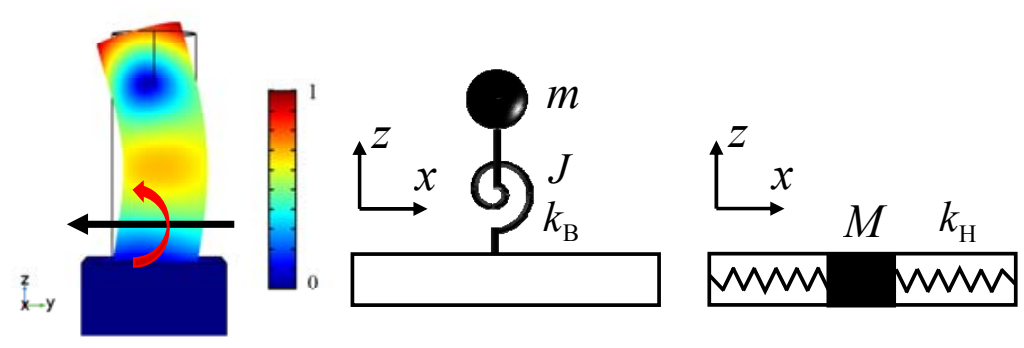

Figure 3: (a) Vertical traction force (left panel) and simplified mass-spring subsystem (right panel) for the compressional resonance C. (b) Scheme of the traction force and moment (left panel), simplified mass-torsional spring subsystem (mid panel) and mass-spring subsystem modelling the bending resonances denoted as A and B in Fig. 1. 
Phys. Rev. Appl. NT10126 - Revised version

\section{DOUBLE-SIDED PILLARED METAMATERIAL}

As discussed in the previous section, the single-sided pillared metamaterial can achieve NMD but not NEM. In order to simultaneously realize both negative properties, we have considered a double-sided pillared metamaterial that, under certain conditions [21,29,31,38], enables enlargement of the width of the low frequency band gap. We show in this section that the double negativity can be obtained through an appropriate choice of the dimensions of the pillar and matrix plate, both of which being made of steel. The unit cell is shown in Fig. 4(a). Two identical pillars are symmetrically arranged on both sides of the matrix plate. The geometric parameters of the unit cell are the same as those of the above single-sided pillared metamaterial. The corresponding band structure is shown in Fig. 4(b). In contrast to the band structure of the single-sided pillared metamaterial, an isolated propagative negative-slope branch arises inside the complete band gap that opens between $3.25 \mathrm{MHz}$ and $3.76 \mathrm{MHz}$. This is almost twice the width of the band gap obtained with the single-sided pillared metamaterial. This enlargement is due to the strong coupling between resonances of the double-sided pillars and Lamb modes in the matrix plate [21].

The isolated branch that ranges from $3.53 \mathrm{MHz}$ to $3.57 \mathrm{MHz}$ divides the band gap into two narrower ones ranging from (in $\mathrm{MHz}$ ) 3.25 to 3.53, and from 3.57 to 3.76 respectively. A zoom in on this propagative branch is displayed in the inset in Fig. 4(b).

(a)

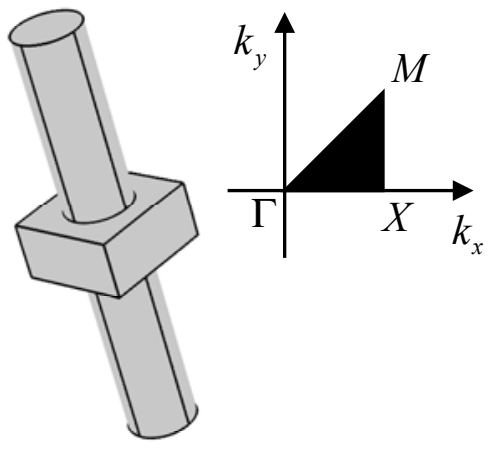

(b)

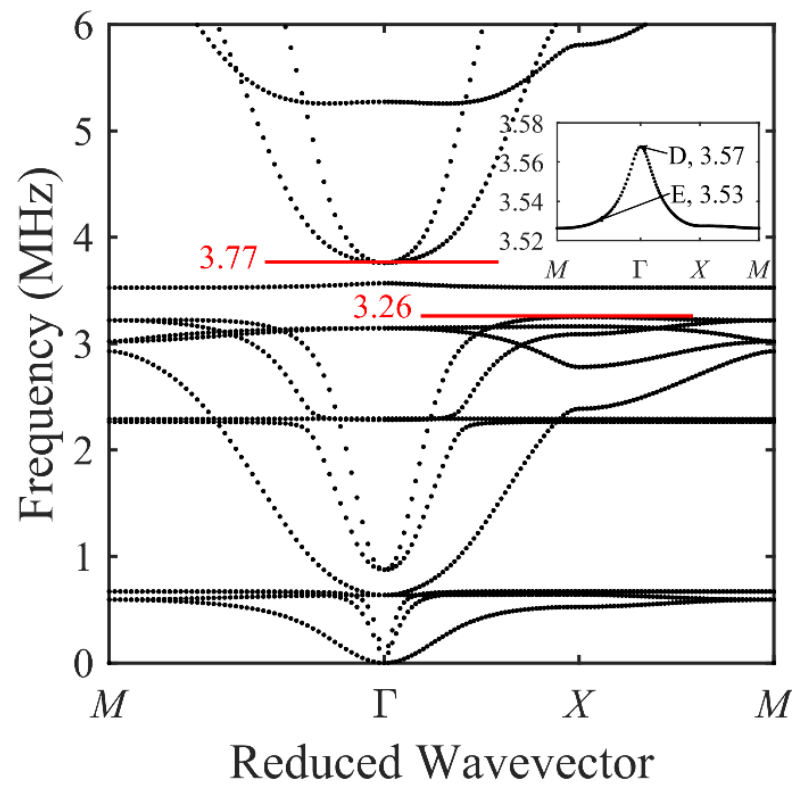


(c)

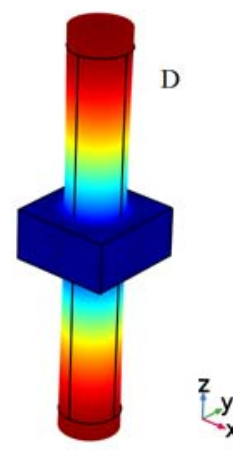

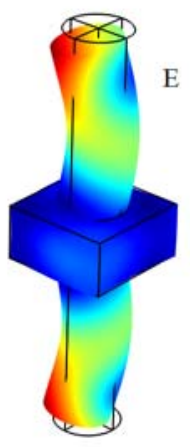

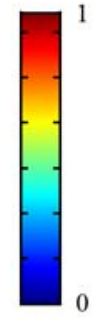

Figure 4: (a) Unit cell of the double-sided pillared metamaterial and first irreducible BZ of the square lattice. (b) Band structure in the low frequency range. Inset: enlargement of the isolated branch. (c) Normalized total displacement field amplitude and deformation in the unit cell at the points denoted as $\mathrm{D}$ and $\mathrm{E}$ in the inset.

The negative slope of this branch throughout the reduced BZ cannot be afforded to a band folding effect and rather suggests simultaneous NMD and NEM resulting from local resonances of the pillars. In support of this argument, we show in Fig. 4(c) the displacement field at two characteristic points on this branch. At point $\Gamma$ in the BZ (point D in the inset in Fig. 4(b)) the displacement field clearly corresponds to a symmetric compressional mode of the pillars whereas the motion of the pillars at midpoint between $\Gamma$ and $X$ is that of a symmetric bending mode (point E in Fig. 4(c)). We show in the next subsection that the former is responsible for NEM whereas the latter, similar to the single-pillared metamaterial, leads to NMD.

\section{A. Formation of the isolated negative-slope branch}

Before analyzing the formation of the isolated branch, it is necessary to identify the resonances in the frequency range of interest. This can be done by computing the displacement field in the unit cell upon the global harmonic excitation $\left(A e^{i \omega t}, 0,0\right)$. On the whole, there are four resonances involving a motion in the plane $(x, z)$, namely: $\mathrm{B}_{\mathrm{A}}(3.17 \mathrm{MHz})$ is the second-order antisymmetric bending resonance; $\mathrm{B}_{\mathrm{S}}(3.26 \mathrm{MHz})$ is the second-order symmetric bending resonance; $\mathrm{C}_{\mathrm{A}}(3.04 \mathrm{MHz})$ is the first-order antisymmetric compressional resonance, and $\mathrm{C}_{\mathrm{S}}(3.57 \mathrm{MHz})$ is the first-order symmetric compressional resonance. The corresponding displacement fields are displayed in Fig. 5(a). Because of the squared symmetry of the unit cell one should also add to this list two bending resonances equivalent to modes $\mathrm{B}_{\mathrm{S}}$ and $\mathrm{B}_{\mathrm{A}}$ respectively, but involving motions along $y$-axis.

As it is the case in the single-sided pillared metamaterial, the formation of the low frequency band gap should be the signature of NMD that results in turn from the combination of the bending and compressional resonances of the two pillars. For verifying this hypothesis, the same formalism as the one 
Phys. Rev. Appl. NT10126 - Revised version

applied in Sec. II-B was implemented to calculate the effective mass density matrix against the frequency. The normalized components $\rho_{11}$ and $\rho_{33}$ displayed in Fig. 5(b), highlight the role played by each resonance. Actually, at the frequencies of modes $\mathrm{B}_{\mathrm{A}}$ and $\mathrm{C}_{\mathrm{S}}$ the changes in $\rho_{11}$ and $\rho_{33}$ are very sharp and cannot cause NMD. On the contrary, both $\rho_{11}$ and $\rho_{33}$ turn negative at the frequencies of $\mathrm{B}_{\mathrm{S}}$ and $\mathrm{C}_{\mathrm{A}}$ which thus directly relate to NMD. The simplified traction forces and moments modeling the pillars at resonances provide a comprehensive understanding of these divergent behaviors. Both are schematically described in Fig. 5(a) by black and red arrows respectively. At $\mathrm{B}_{\mathrm{A}}$ resonance, the moments induced by the two identical pillars are the same whereas the traction forces are pointing in opposite directions and hence the resultant force is zero. Although the resultant moment is not equal to zero, it does not couple with the motion in the plate and this bending resonance cannot induce NMD: $\rho_{11}$ and $\rho_{22}$ do not turn negative around the antisymmetric bending resonance, except at the exact resonance frequency. The reverse phenomenon occurs at the frequency of $B_{S}$. Actually at this frequency the resultant moment is equal to zero, but not the resultant traction force that consequently contributes to the negative value of $\rho_{11}$ in between $3.26 \mathrm{MHz}$ and $3.77 \mathrm{MHz}$. By symmetry, $\rho_{22}$ gets also negative in the same interval.

The same argument applies for both compressional modes. Indeed, the resultant force at the frequency of $\mathrm{C}_{\mathrm{S}}$ mode (i.e. $3.57 \mathrm{MHz}$ ) is null and this mode does not trigger negative values of the component $\rho_{33}$. Conversely, the situation at the frequency of $\mathrm{C}_{\mathrm{A}}$ is similar to the one observed at the compressional resonance in the single-sided pillared metamaterial: the axial forces in the pillars combine and $\rho_{33}$ takes negative values in the range $3.04 \mathrm{MHz}$ to $5.29 \mathrm{MHz}$. 
(a)
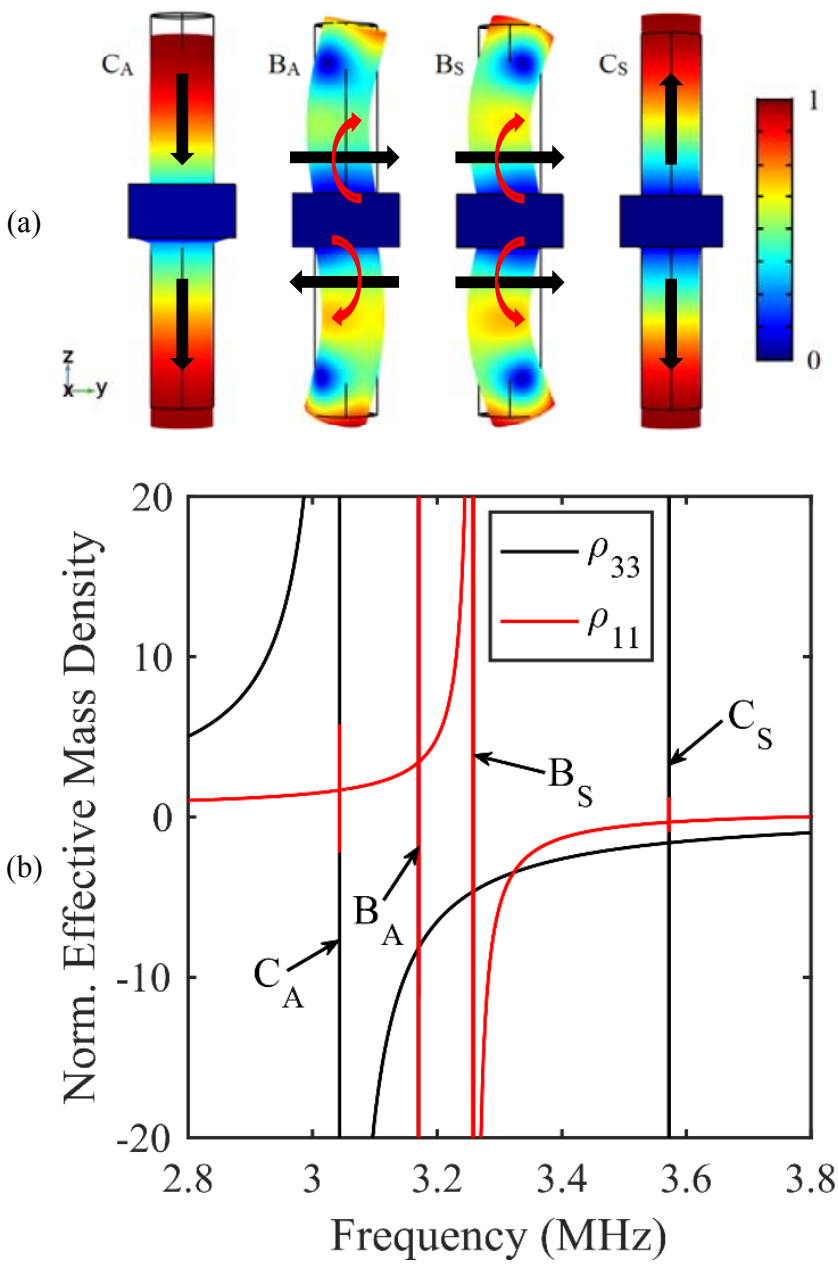

Figure 5: (a) Normalized total displacement field amplitude and deformation of the unit cell at four representative resonance frequencies upon excitation $\left(A e^{i \omega t}, 0,0\right)$. (b) Components of the effective mass density $\rho_{11}$ (red line) and $\rho_{33}$ (black line) against the excitation frequency.

Overall, NMD is achieved at any frequency in the interval stretching from $3.26 \mathrm{MHz}$ to $3.77 \mathrm{MHz}$, including the vibrational modes on the isolated branch therein, where $\rho_{11}, \rho_{22}$, and $\rho_{33}$ are all negative as shown in Fig. 5(b). Both these values exactly match the limits of the low frequency band gap displayed in Fig. 4(b) and further highlighted with red lines in Fig. 4(b).

Although the isolated branch is relatively flat, especially along $X M$ in the BZ, the group velocity is nonnull and the propagation of elastic energy at the corresponding frequencies is allowed. Therefore, the dynamic elastic modulus is necessarily negative for any vibrational mode on this branch. Figure 5(b) clearly shows that the narrow interval around $3.57 \mathrm{MHz}$ where propagation is allowed corresponds to the first-order symmetric compressional resonance $\mathrm{C}_{\mathrm{S}}$ which is thus causing NEM and in turn the doubly negative property. It should be noticed however that this resonance involves a purely out-of-plane motion 
only at points $\Gamma, X$ and $M$ of the BZ. In between these main points in the BZ, in-plane motions contribute also to the deformation of the pillars, as it can be observed in Fig. 4(c), point E.

\section{B. Occurrence of the negative elastic modulus}

Similar to the analytical study developed above to explain NMD achieved by the single-sided pillared metamaterial, one can model the symmetric compressional vibration of the double-sided pillared system as two mass-spring subsystems, each featuring a mass $m$ and a stiffness $k_{\mathrm{C}}$, vibrating in phase as shown in Fig. 6(a). In this figure, $e$ is the thickness of the matrix plate; $A_{x}=e \times a$ and $A_{z}=a^{2}$ are the areas of boundaries parallel to the planes $(y, z)$ and $(x, y)$ respectively. To calculate the traction forces exerted on the plate when the pillars are on a vertical motion, let us consider two harmonic tension forces $\mathbf{F}_{\mathrm{x}}$ with the same amplitude, both applied along $x$-axis on the lateral boundaries parallel to the plane $(y, z)$ and pointing in opposite directions (Fig. 6(a)). Displacements $\pm x_{1}$ of the lateral boundaries, $\pm z_{1}$ of the free surfaces and $\pm z_{2}$ of mass $m$ result from the forces $\mathbf{F}_{\mathbf{x}}$ (Fig. 6(b)). It is expected that the harmonic deformation induced by $\mathbf{F}_{\mathbf{x}}$ allows to excite the resonances of the mass-spring subsystems if the frequency is properly tuned.

(a)

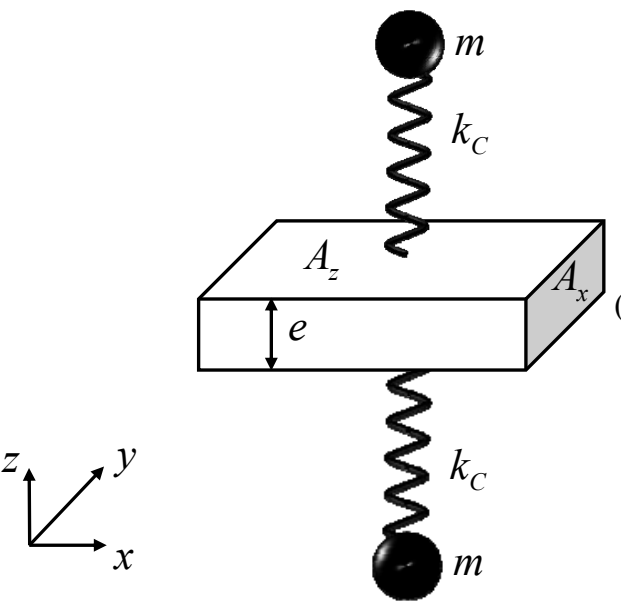

(b) $F_{x}$

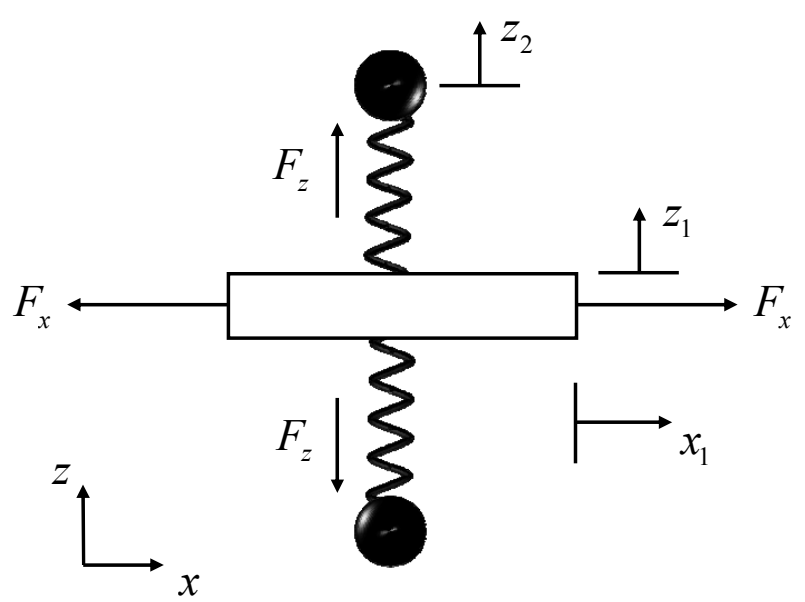

Figure 6: (a) Model of the unit cell with two mass-spring subsystems. (b) Side view of the unit cell and notations of displacements induced by the forces applied along $x$-axis.

To simplify the derivation of the effective elastic modulus $E_{\text {eff }}$ of the unit cell, we assume that the reactive forces $\mathbf{F}_{\mathbf{z}}$ caused by the vibration of the mass-spring subsystems are uniformly distributed on the matrix plate. Under this hypothesis, the dynamic stresses tensor $\boldsymbol{\sigma}$ and strains tensor $\boldsymbol{\varepsilon}$ in the matrix plate submitted to a harmonic excitation are given by Hooke's law and can be expressed as: 
Phys. Rev. Appl. NT10126 - Revised version

$$
\left\{\begin{array}{l}
\sigma_{\mathrm{x}}=\frac{F_{\mathrm{x}}}{A_{\mathrm{x}}} \\
\sigma_{\mathrm{y}}=0 \\
\sigma_{\mathrm{z}}=\frac{F_{\mathrm{z}}}{A_{\mathrm{z}}} \\
\varepsilon_{\mathrm{x}}=\frac{2 x_{1}}{a}=\frac{1}{E}\left(\sigma_{\mathrm{x}}-v \sigma_{\mathrm{z}}\right) \\
\varepsilon_{\mathrm{z}}=\frac{2 z_{1}}{e}=\frac{1}{E}\left(\sigma_{\mathrm{z}}-v \sigma_{\mathrm{x}}\right)
\end{array}\right.
$$

As previously, $E$ is the elastic modulus and $v$ the Poisson's ratio. On the other hand, the equation of motion of the mass-spring subsystems is:

$$
m \frac{\partial^{2} z_{2}}{\partial t^{2}}=-F_{z}=-k_{C}\left(z_{2}-z_{1}\right)
$$

By combining Eqs. (7) and (8) one easily obtains:

$$
\frac{F_{\mathrm{x}}}{A_{\mathrm{x}}}=E\left(1-\frac{v^{2}}{1+\frac{k_{\mathrm{M}}}{k_{\mathrm{C}}} \frac{\omega^{2}-\omega_{0}^{2}}{\omega^{2}}}\right)^{-1} \frac{2 x_{1}}{a}
$$

where $\omega_{0}=\left(k_{\mathrm{C}} / \mathrm{m}\right)^{1 / 2}$ is the natural frequency of the mass-spring subsystem, $k_{\mathrm{M}}=2 E A_{\mathrm{z}} / \mathrm{e}$ is the effective stiffness of the plate.

Equation (9) is nothing else but the Hook's law applied to an effective medium which effective elastic modulus $E_{\text {eff }}=E\left(1-\frac{v^{2}}{1+\frac{1}{\eta} \frac{\omega^{2}-\omega_{0}^{2}}{\omega^{2}}}\right)^{-1}$ depends on the frequency $\omega / 2 \pi$ and takes into account the internal motions of the unit cell through the parameters $\eta=k_{\mathrm{C}} / k_{\mathrm{M}}$ and $\omega_{0}$.

The effective elastic modulus $E_{\text {eff }}$ can also be written evidently as:

$$
E_{e f f}=E\left(1+\frac{v^{2} \omega^{2}}{\left(1-v^{2}+1 / \eta\right) \omega^{2}-1 / \eta \omega_{0}^{2}}\right)
$$


The effective elastic modulus diverges if $\omega=\omega_{0}\left(\frac{1}{\left(1-v^{2}\right) \eta+1}\right)^{1 / 2}$, which thus must be regarded as the resonant frequency of the effective material. It is interesting to notice here that the resonant frequency of the effective material is not that of the mass-spring subsystem. Furthermore, $E_{\text {eff }}=0$ when $\omega=\omega_{0}(1 / \eta+1)^{1 / 2}$ and $E_{e f f}$ is negative if:

$$
\omega_{0}\left(\frac{1}{\eta+1}\right)^{1 / 2}<\omega<\omega_{0}\left(\frac{1}{\left(1-v^{2}\right) \eta+1}\right)^{1 / 2} .
$$

This interval is all the more broad that the Poisson's ratio $v$ is large. Although this simplified model hardly allows predicting the position and width of the isolated double-negative branch, it provides an intuitive comprehension of the occurrence of NEM and in turn of the double negativity of the double-sided pillared metamaterials.

\section{Evolution of the two band gaps and isolated branch vs the geometric parameters of the unit cell}

Being the consequence of a resonant phenomenon, the double-negative branch of the proposed structure is relatively narrow which may be a drawback for some applications. However, the width of the frequency band where double negativity occurs can be increased through a proper choice of the geometrical parameters of the unit cell [12]. We investigate in this section the influence of the height and diameter of the pillar as well as the thickness of the matrix plate, on both the width of the forbidden band gap and the negative-slope branch.

Both Figs. 7(a) and 7(b) show the effect of the dimensions of the pillars on both the low frequency band gap and the negative-slope branch. Increasing the height of the pillar leads to the decrease of the central frequency of the band gap, as well as to the decrease of the range where the double negativity occurs. This should be related to the decrease of the compressional resonance frequency for increasing height of the pillars [26]. Moreover, the lower part of the band gap broadens as the height of the pillar increases. In contrast, the propagative branch moves closer to the upper limit of the band gap as the height of the pillar increases leading to the closure of the upper part when the height of each pillar is more than about $350 \mu \mathrm{m}$. Remembering that the effective mass density tends towards zero while keeping negative values when the frequency approaches the upper limit of the band gap (see Fig. 5(b)), it is expected that this structure may behave as a zero-index elastic metamaterial. Actually, in that case the phase velocity in the metamaterial tends to infinity and therefore the refractive index (i.e. ratio of the velocity in the background to the velocity in the metamaterial) goes to zero. This point is further developed below. 
Phys. Rev. Appl. NT10126 - Revised version

On the other hand, the bending resonance is very sensitive to the diameter $d$ of the pillars [26] and consequently this parameter has a large impact on the width of the band gap that broadens as the diameter increases (Fig. 7(b)). However, the width of the double-negative band is very little affected by this parameter and remains equal to about $8.5 \%$ of the width of the forbidden band whatever the diameter of the pillars is.

(a)
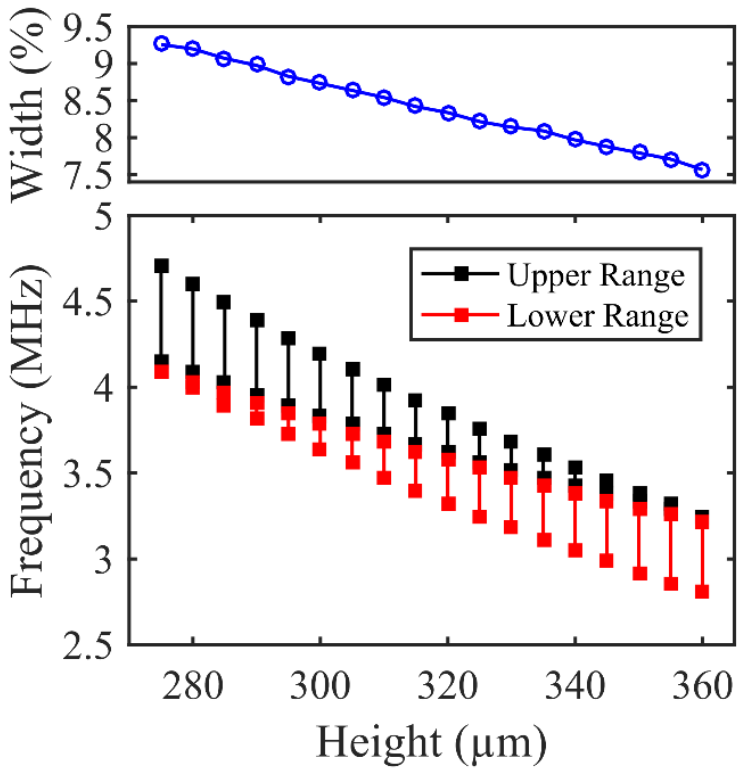

(b)
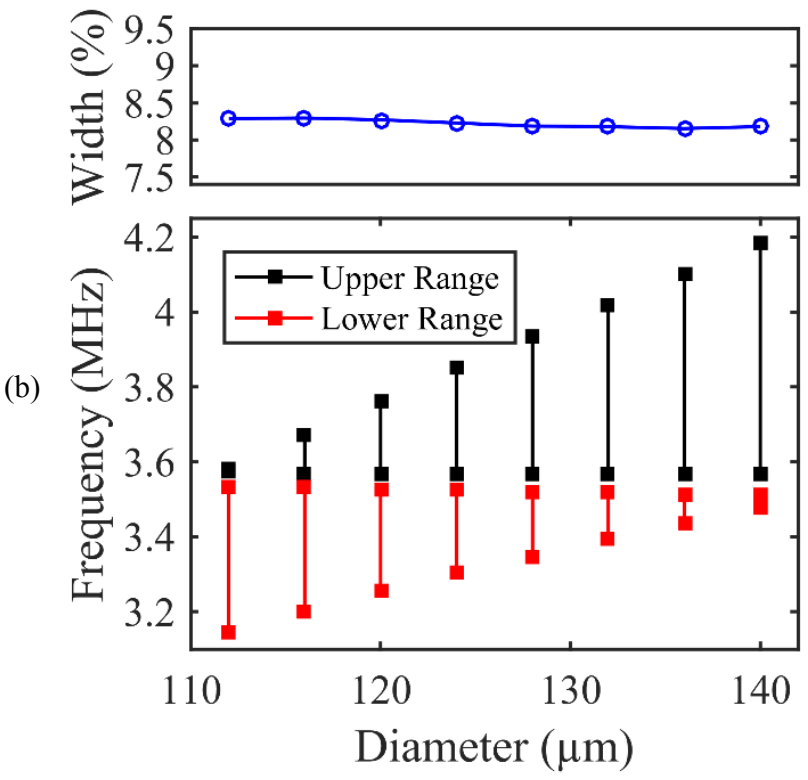

Figure 7: Edges of the band gap (lower panel) and relative width of the double-negative band (upper panel) against (a) the height and (b) the diameter of the pillars. The black (resp. red) solid lines stand for the upper (resp. lower) part of the band gap. In both panels the blue curve is the relative width of the band where the double negativity occurs. 
However, the parameter that most efficiently affects the width of both the band gap and the propagative branch is the thickness of the plate (Fig. 8). Actually, both parts of the band gap slightly decrease as the thickness increases while at the same time the width $\Delta \omega$ of the double negativity band linearly increases with increasing thickness of the plate. The relative width of the band exhibiting NEM reaches the maximum value of $\sim 20 \%$ of the total band gap when $e=180 \mu \mathrm{m}$.

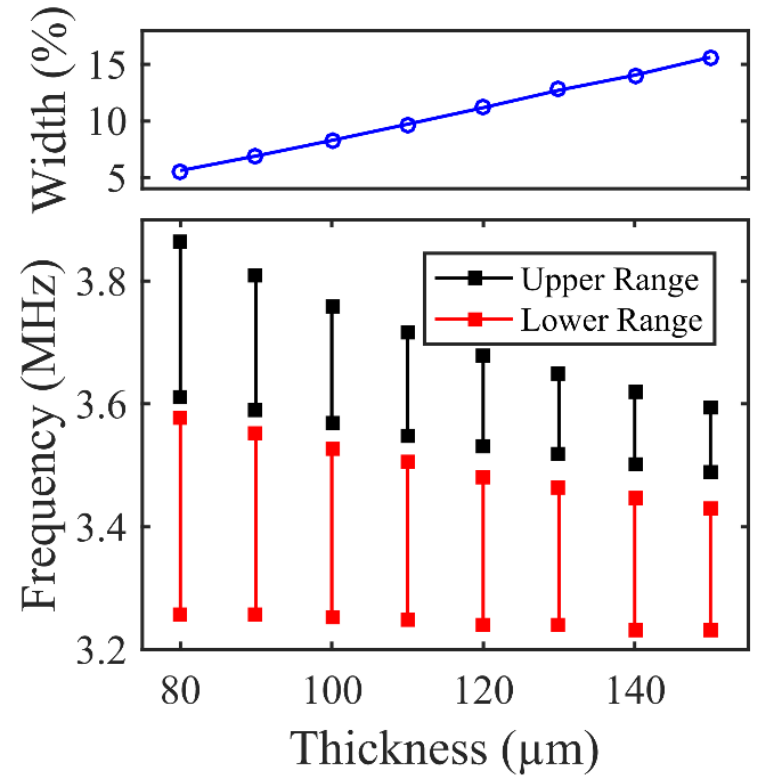

Figure 8: Edges of the band gap (lower panel) and relative width of the double-negative band (upper panel) against the thickness of the plate. The black (resp. red) solid lines stand for the upper (resp. lower) part of the band gap. The blue curve is the relative width of the band where double negativity occurs.

A simple model allows to summarize these results. Actually, at $\mathrm{C}_{\mathrm{S}}$ resonance there is almost no displacement at the foot of the pillars (see Fig. 5(a)) that can be modelled as a mass-spring system with a steady attachment point and for which the displacement on top of the pillar is proportional to the axial force. With these assumptions, Hook's law leads to $k_{\mathrm{C}}=\pi d^{2} E / 4 h$ and the ratio $\eta=k_{\mathrm{C}} / k_{\mathrm{M}}$ can be written as $\eta=\pi d^{2} e / 8 h a^{2}$. With the typical dimensions of the unit cell, $\eta$ is always much less than unity and Eq. (11) can be expanded as:

$$
\Delta \omega=\frac{\pi}{16} v^{2}(E / \rho)^{1 / 2} \frac{d^{2} e}{h^{2} a^{2}},
$$

where we have used $\omega_{0}=\left(k_{\mathrm{C}} / \mathrm{m}\right)^{1 / 2}=\left(E / \rho h^{2}\right)^{1 / 2}$. Equation (12) gives full account of the behaviors displayed in Fig. 7 and Fig. 8. 
Phys. Rev. Appl. NT10126 - Revised version

Although relatively narrow even when it is maximum, the width of the doubly negative band remains sufficient for an experimental validation based onto conventional techniques as for instance the excitation of Lamb waves with interdigitated transducers (IDT) on a piezoelectric film. Actually, in the frequency range that we have considered, elastic waves featuring a spectral purity $\delta f$ better than the width of the NEM band [39] can be easily obtained with an IDT comprising only a few tens pairs of electrodes [40,41], allowing in turn to tailor the transition from single negativity to double negativity. It is worth noting, however, that our results are not specific to the $\mathrm{MHz}$ range and may be transposed either to the $\mathrm{GHz}$ or the $\mathrm{kHz}$ range by rescaling both the thickness of the plate and the lattice constant while keeping the ratio $\lambda / \mathrm{a}=$ const ( $\lambda$ is the wavelength at the operating frequency) and adapting the dimensions of the pillars accordingly. The high frequency domain fits with nanostructures whose typical dimensions (thickness of the plate, high and diameter of the pillars, and lattice constant) are of a few micrometers or less, with possible applications such as the vibration isolation of sensitive components, the super-focusing, the highresolution imaging, or the cloaking of elastic waves (see below). At the other end of the scale, doublesided pillared metamaterials with typical dimensions in the millimeter range are excellent candidates for applications for noise reduction and filtering in the audio range. However, a narrow band is not necessarily a drawback and, even it becomes an advantage for all applications and devices where a high selectivity in frequency is a requirement.

\section{Negative refraction of Lamb waves and cloaking}

The negative refraction (NR) of elastic waves through the interface between a phononic crystal and the adjacent medium, basically relates to the bands folding resulting from the periodicity, and to the negative slope of some dispersion branches [42-44]. As a result NR in these heterostructures only occurs when the wavelength is of the same order of magnitude as the period and double-negative elastic properties are not relevant to explain this behavior. In contrast, in an elastic metamaterial the negative refraction is the direct consequence of simultaneous NMD and NEM and as such, occurs in the long wavelength limit [24]. The occurrence of NR would provide therefore further evidence of the double negativity in the double-sided pillared metamaterial. For this purpose, we have considered the FEM model shown in Fig. 9(a). This figure displays a 2D prism shaped metamaterial constituted by 120 unit cells arranged in a $45^{\circ}$ isosceles triangle within a circular steel plate. The plate was surrounded by a perfectly matched layer (PML) layer to eliminate reflections from the boundaries. The two perpendicular sides of the prism were set parallel to the lattice directions $\Gamma X$ and $X M$. A line source $1600 \mu \mathrm{m}$ long was placed $100 \mu \mathrm{m}$ away from the inlet interface perpendicularly to $\Gamma X$ direction. Lamb waves either symmetric or antisymmetric, were normally incident from the left and were propagating along $\Gamma X$ direction. The frequency of the exciting waves was set to the mid value of the peak in the isolated branch, namely $3.55 \mathrm{MHz}$. At this frequency, the 
Phys. Rev. Appl. NT10126 - Revised version

wavelength of the symmetric (resp. antisymmetric) Lamb mode is about eight (resp. three) times the lattice constant.

The in-plane velocity field for the symmetric excitation is plotted in Fig. 9(b). It is clear from this plot that the symmetric Lamb mode propagates in the metamaterial and gets negatively refracted at the outlet interface. This result demonstrates that both resonances $B_{S}$ (related to NMD) and $C_{S}$ (related to NEM) are simultaneously excited by the Lamb mode, allowing in turn the propagation in the metamaterial. One should also notice that, as a resonant process, even the small out-of-plane component of the Lamb mode is able to excite the symmetric compressional mode $C_{S}$. When NR results from the band folding at frequencies larger than the Bragg gap, it is generally accompanied with a high level of reflection at each interface between the phononic crystal and the background, which may constitutes a severe drawback in many applications. It is worth mentioning that this is not the case here. To show this, we have computed the transmission coefficient of a symmetric Lamb wave impinging at normal incidence a structure made of eleven unit cells along $x$-axis and infinite along the $y$-direction. The result displayed in Fig. 9(c) shows that the transmission is equal to unity in the whole band where double negativity occurs.

The result is totally different with the antisymmetric mode, as can be seen from Fig. 9(d). Actually, for this polarization the wave cannot propagate into the metamaterial and is totally reflected at the inlet interface. This comes from the symmetry of this mode that cannot couple neither to the symmetric compressional mode $\mathrm{C}_{\mathrm{S}}$ nor to the symmetric bending mode $\mathrm{B}_{\mathrm{S}}$ of the double-sided pillars and therefore prevents the occurrence of the necessary double negativity for the propagation to be allowed. 
(a)

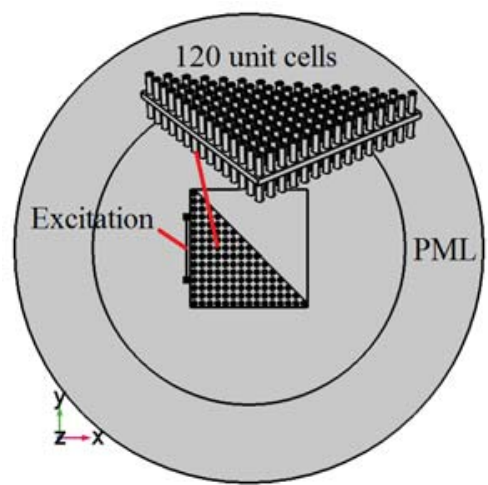

(c)

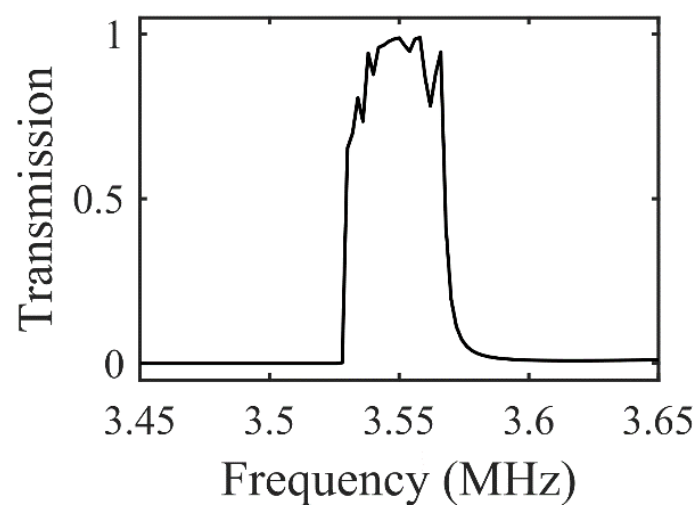

(b)

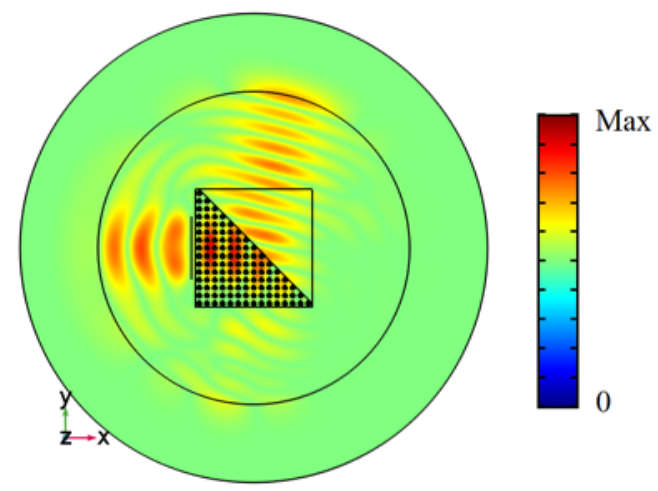

(d)

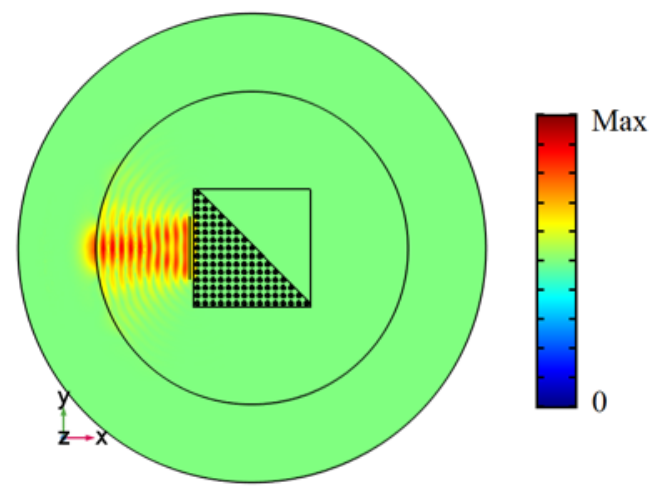

Figure 9: (a) Finite element model used to demonstrate the negative refraction of Lamb waves; (b) Inplane velocity plot under symmetric excitation at $3.55 \mathrm{MHz}$; (c) Transmission coefficient of a symmetric Lamb wave impinging the phononic crystal at normal incidence; (d) In-plane velocity for an antisymmetric excitation at $3.55 \mathrm{MHz}$.

Another current topic is the implementation of acoustic cloaking based on a zero-index metamaterial and much work has been devoted to this research since a few years [9,45-48]. This exciting phenomenon can be ascribed to the effective phase velocity that tends towards infinity if the effective mass density is close to zero [45]. Therefore, an alternative method to obtain a refractive index with a null value consists in combining an infinite effective elastic modulus to a finite effective mass density. As discussed above, the symmetric compressional resonance $\mathrm{C}_{\mathrm{S}}$ in the double-sided pillared metamaterial causes NEM with an infinite value when the excitation frequency is equal or quite close to $3.57 \mathrm{MHz}$, while the effective density keeps a negative but still finite value at this frequency; zero-index behavior should therefore be observed at this frequency. To check this assumption, we have computed the displacement field in the numerical sample displayed in the top panel in Fig. 10. The metasurface is made of $21 \times 11$ unit cells and features a $7 a \times 3 a$ void in its center. A zero-order symmetric Lamb wave is excited at a distance of $1 \mathrm{~mm}$ from the left edge of the metamaterial and PML's are implemented on each side of the sample to eliminate any reflection from the boundaries. Periodic boundary conditions are applied on the other two edges. The displacement field when the frequency is tuned to $3.57 \mathrm{MHz}$ is shown in the mid panel of Fig. 10. At this 
frequency, the wave front keeps plane upon transmission through the sample, except around the void where scattering effects are observable. This is a clear confirmation that, as a result of the nearly infinite phase velocity in the metamaterial, the phase of the symmetric Lamb wave keeps a constant value during propagation, allowing for a cloaking effect in this system. In contrast, when the working frequency is tuned to $4 \mathrm{MHz}$, i.e. a frequency where the elastic modulus is positive, the Lamb wave undergoes strong scattering on the void, giving rise to the distorted wave front observable in the bottom panel of Fig. 10. This simple analysis of the transmission through the metasurface unambiguously shows that the shielding of substructures at specific frequencies may be achieved with this geometry.

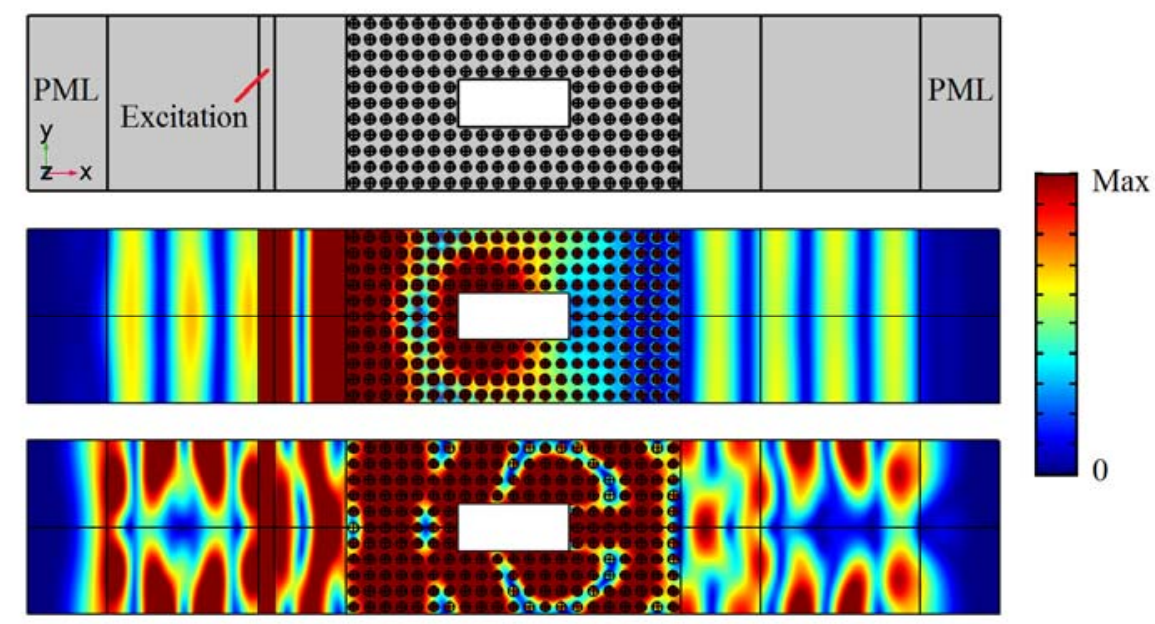

Figure 10: Finite element model (top panel) used to bear out the cloaking effect and total displacement field upon symmetric excitation at $3.57 \mathrm{MHz}$ (middle panel) and $4 \mathrm{MHz}$ (bottom panel)

\section{CONCLUSION}

In this work, we have numerically investigated the negative properties of a single-phase double-sided pillared metamaterial. In contrast to the single-sided pillared structure where NEM never occurs, an isolated propagative branch with negative slope appears in the band structure of the proposed system. Symmetric Lamb wave at a frequency in this branch can propagate within the metamaterial whereas the propagation of the antisymmetric Lamb mode is forbidden. Comparisons with the case of the single-sided pillared system well explains the formation of the low frequency band gap and the occurrence of the double negativity. Actually, in the single-sided pillared structure the negative effective mass density results from the combination of both the bending and the compressional resonances of the pillar which together allow for the formation of the band gap. At resonance, the bending motion may be broken down into a rotational and a translational motion. The latter causes the effective mass density to be negative, whereas the moment applied to the plate by the rotational motion is ineffective. Afterwards, the doublesided pillared metamaterial is investigated. The mechanism responsible for the occurrence of the effective 
Phys. Rev. Appl. NT10126 - Revised version

mass density and elastic modulus simultaneously negative is discussed in details. It is shown that the negative effective mass density is achieved owing to the combination of the symmetric bending resonance and the antisymmetric compressional resonance of the pillars. It is further shown that the negative effective elastic modulus relates to their symmetric compressional resonance. In other words, the doublesided pillared structure has the advantage to allow to separate the symmetric and antisymmetric modes which is not achievable with the single-sided pillared structure. The symmetric band is created by the close vicinity of a bending resonance and a compressional resonance that together allow for the opening of a narrow pass band.

Furthermore, the influence of the geometrical parameters of the unit cell on the width of both the band gap and the double-negative band is studied. The band gap shifts downwards when increasing the height of the pillar or decreasing the diameter of the pillar, while the thickness of the plate weakly affects the position of the band gap. More importantly, both the height of the pillars and the thickness of the matrix plate have a strong impact on the width of the double-negative band. By carefully designing the unit cell, the width can be significantly enlarged. In particular, equation (11) demonstrates that materials with large Poisson's ratio allow for larger width. Finally, two properties which both directly ensue from the double negativity of the structure, namely the negative refraction and zero-index refraction of Lamb waves in the long wavelength regime, are shown to take place when the frequency lies in the double-negative band. While the central focus of this study was the demonstration of the simultaneous negativity of both the effective mass density and elastic modulus in the pillared metamaterials, the occurrence of a refractive index with a zero value, even in the absence of a Dirac-like cone at the center of the BZ [20], constitutes a significant fallout of this work.

\section{Acknowledgements}

Wei Wang acknowledges the research scholarship No. 201608310130 provided by the China Scholarship Council.

\section{References}

[1] T.T. Wu, Z.G. Huang, T.C. Tsai, and T.C. Wu, Evidence of complete band gap and resonances in a plate with periodic stubbed surface, Appl. Phys. Lett. 93, 111902 (2008).

[2] S. Yao, X. Zhou, and G. Hu, Experimental study on negative effective mass in a 1D mass-spring system, New J. Phys. 10, 043020 (2008).

[3] H.H. Huang and C.T. Sun, Wave attenuation mechanism in an acoustic metamaterial with negative effective mass density, New J. Phys. 11, 013003 (2009).

[4] R. Zhu, X.N. Liu, G.L. Huang, H.H. Huang, and C.T. Sun, Microstructural design and experimental 
Phys. Rev. Appl. NT10126 - Revised version

validation of elastic metamaterial plates with anisotropic mass density, Phys. Rev. B 86, 144307 (2012).

[5] N. Fang, D. Xi, J. Xu, M. Ambati, W. Srituravanich, C. Sun, and X. Zhang, Ultrasonic metamaterials with negative modulus, Nat. Mater. 5, 452 (2006).

[6] S.H. Lee, C.M. Park, Y.M. Seo, Z.G. Wang, and C.K. Kim, Composite acoustic medium with simultaneously negative density and modulus, Phys. Rev. Lett. 104, 054301 (2010).

[7] M. Yang, G. Ma, Z. Yang, and P. Sheng, Coupled membranes with doubly negative mass density and bulk modulus, Phys. Rev. Lett. 110, 134301 (2013).

[8] R. Zhu, X.N. Liu, G.K. Hu, C.T. Sun, and G.L. Huang, Negative refraction of elastic waves at the deep-subwavelength scale in a single-phase metamaterial., Nat. Commun. 5, 5510 (2014).

[9] H.-W. Dong, S.-D. Zhao, Y.-S. Wang, and C. Zhang, Topology optimization of anisotropic broadband double-negative elastic metamaterials, J. Mech. Phys. Solids 105, 54 (2017).

[10] D. Zhang, J. Zhao, B. Bonello, F. Zhang, W. Yuan, Y. Pan, and Z. Zhong, Investigation of surface acoustic wave propagation in composite pillar based phononic crystals within both local resonance and Bragg scattering mechanism regimes, J. Phys. D. Appl. Phys. 50, 435602 (2017).

[11] Y. Ding, Z. Liu, C. Qiu, and J. Shi, Metamaterial with simultaneously negative bulk modulus and mass density, Phys. Rev. Lett. 99, 093904 (2007).

[12] Y. Jin, B. Bonello, R.P. Moiseyenko, Y. Pennec, O. Boyko, and B. Djafari-Rouhani, Pillar-type acoustic metasurface, Phys. Rev. B 96, 104311 (2017).

[13] Z. Liu, X. Zhang, Y. Mao, Y.Y. Zhu, Z. Yang, C.T. Chan, and P. Sheng, Locally resonant sonic materials, Science 289, 1734 (2000).

[14] X.N. Liu, G.K. Hu, G.L. Huang, and C.T. Sun, An elastic metamaterial with simultaneously negative mass density and bulk modulus, Appl. Phys. Lett. 98, 251907 (2011).

[15] J.H. Oh, Y.E. Kwon, H.J. Lee, and Y.Y. Kim, Elastic metamaterials for independent realization of negativity in density and stiffness, Sci. Rep. 6, 23630 (2016).

[16] Y. Pennec, B. Djafari-Rouhani, H. Larabi, J.O. Vasseur, and A.C. Hladky-Hennion, Low-frequency gaps in a phononic crystal constituted of cylindrical dots deposited on a thin homogeneous plate, Phys. Rev. B 78, 104105 (2008).

[17] V.E. Gusev and O.B. Wright, Double-negative flexural acoustic metamaterial, New J. Phys. 16, 123053 (2014).

[18] H. Zhu and F. Semperlotti, Phononic thin plates with embedded acoustic black holes, Phys. Rev. B 91, 104304 (2015).

[19] H. Zhu and F. Semperlotti, Anomalous Refraction of Acoustic Guided Waves in Solids with Geometrically Tapered Metasurfaces, Phys. Rev. Lett. 117, 034302 (2016).

[20] H. Zhu and F. Semperlotti, Double-Zero-Index Structural Phononic Waveguides, Phys. Rev. Appl. 8, 
Phys. Rev. Appl. NT10126 - Revised version

064031 (2017).

[21] M. B. Assouar and M. Oudich, Enlargement of a locally resonant sonic band gap by using doublesides stubbed phononic plates, Appl. Phys. Lett. 100, 123506 (2012).

[22] S. Benchabane, R. Salut, O. Gaiffe, V. Soumann, M. Addouche, V. Laude, and A. Khelif, SurfaceWave Coupling to Single Phononic Subwavelength Resonators, Phys. Rev. Appl. 8, 034016 (2017).

[23] O.R. Bilal and M.I. Hussein, Trampoline metamaterial: Local resonance enhancement by springboards, Appl. Phys. Lett. 103, 111901 (2013).

[24] M. Oudich, B. Djafari-Rouhani, Y. Pennec, M.B. Assouar, and B. Bonello, Negative effective mass density of acoustic metamaterial plate decorated with low frequency resonant pillars, J. Appl. Phys. 116, 184504 (2014).

[25] M. Oudich, B. Djafari-Rouhani, B. Bonello, Y. Pennec, S. Hemaidia, F. Sarry, and D. Beyssen, Rayleigh Waves in Phononic Crystal Made of Multilayered Pillars: Confined Modes, Fano Resonances, and Acoustically Induced Transparency, Phys. Rev. Appl. 9, 034013 (2018).

[26] O.M. Ramahi, T.S. Almoneef, M. AlShareef, and M.S. Boybay, Metamaterial particles for electromagnetic energy harvesting, Appl. Phys. Lett. 101, 173903 (2012).

[27] M. Carrara, M.R. Cacan, J. Toussaint, M.J. Leamy, M. Ruzzene, and A. Erturk, Metamaterialinspired structures and concepts for elastoacoustic wave energy harvesting, Smart Mater. Struct. 22, 065004 (2013).

[28] D. Royer and E. Dieulesaint, Elastic Waves in Solids (Springer-Verlag, Berlin Heidelberg, 2000).

[29] S. Li, T. Chen, X. Wang, Y. Li, and W. Chen, Expansion of lower-frequency locally resonant band gaps using a double-sided stubbed composite phononic crystals plate with composite stubs, Phys. Lett. A 380, 2167 (2016).

[30] M. B. Assouar, M. Senesi, M. Oudich, M. Ruzzene, and Z. Hou, Broadband plate-type acoustic metamaterial for low-frequency sound attenuation, Appl. Phys. Lett. 101, 173505 (2012).

[31] A. Hu, X. Zhang, F. Wu, and Y. Yao, Enlargement of the locally resonant Lamb wave band gap of the phononic crystal plate at the deep sub-wavelength scale, Mater. Res. Express 1, 045801 (2014).

[32] M. Oudich, Y. Li, B.M. Assouar, and Z. Hou, A sonic band gap based on the locally resonant phononic plates with stubs, New J. Phys. 12, 083049 (2010).

[33] R. Pourabolghasem, A. Khelif, S. Mohammadi, A.A. Eftekhar, and A. Adibi, Physics of band-gap formation and its evolution in the pillar-based phononic crystal structures, J. Appl. Phys. 116, 013514 (2014).

[34] S.H. Lee and O.B. Wright, Origin of negative density and modulus in acoustic metamaterials, Phys. Rev. B 93, 024302 (2016).

[35] R. Zhu, M. Reynolds, and G.L. Huang, Numerical effective formulation for guided wave propagation 
Phys. Rev. Appl. NT10126 - Revised version

in a metamaterial plate with anisotropic mass density, Health Monitoring of Structural and Biological Systems, Proc. of SPIE Vol. 8348, 83481X (2012).

[36] Y. Xiao, J. Wen, and X. Wen, Flexural wave band gaps in locally resonant thin plates with periodically attached spring-mass resonators, J. Phys. D. Appl. Phys. 45, 195401 (2012).

[37] D. Qian and Z. Shi, Bandgap properties in simplified model of composite locally resonant phononic crystal plate, Phys. Lett. A 381, 3505 (2017).

[38] C. Ma, J. Guo, and Y. Liu, Extending and lowing band gaps in one-dimensional phononic crystal strip with pillars and holes, J. Phys. Chem. Solids 87, 95 (2015).

[39] The bandwidth at $3 \mathrm{~dB}$ of a transducer comprising $\mathrm{N}$ pairs of electrodes is given by $\delta f=0.885 / N f_{0}$; see for example [28].

[40] R.S.C. Monkhouse, P.D. Wilcox, and P. Cawley, Flexible interdigital PVDF transducers for the generation of Lamb waves in structures, Ultrasonics 35, 489 (1997).

[41] M. Castaings, R.S.C. Monkhouse, M.J.S. Lowe, and P. Cawley, The performance of flexible interdigital PVDF lamb wave transducers, Acta Acust. Acust. 85, 842 (1999).

[42] J. Pierre, O. Boyko, L. Belliard, J.O. Vasseur, and B. Bonello, Negative refraction of zero order flexural Lamb waves through a two-dimensional phononic crystal, Appl. Phys. Lett. 97, 121919 (2010).

[43] A. Sukhovich, L. Jing, and J.H. Page, Negative refraction and focusing of ultrasound in twodimensional phononic crystals, Phys. Rev. B 77, 014301 (2008).

[44] J. Bucay, E. Roussel, J.O. Vasseur, P.A. Deymier, A.C. Hladky-Hennion, Y. Pennec, K. Muralidharan, B. Djafari-Rouhani, and B. Dubus, Positive, negative, zero refraction, and beam splitting in a solid/air phononic crystal: Theoretical and experimental study, Phys. Rev. B 79, 214305 (2009).

[45] R. Graciá-Salgado, V.M. García-Chocano, D. Torrent, and J. Sánchez-Dehesa, Negative mass density and $\rho$-near-zero quasi-two-dimensional metamaterials: Design and applications, Phys. Rev. B 88, 224305 (2013).

[46] B. Edwards, A. Alù, M.E. Young, M. Silveirinha, and N. Engheta, Experimental Verification of Epsilon-Near-Zero Metamaterial Coupling and Energy Squeezing Using a Microwave Waveguide, Phys. Rev. Lett. 100, 033903 (2008).

[47] M. Silveirinha and N. Engheta, Tunneling of Electromagnetic Energy through Subwavelength Channels and Bends using $\varepsilon$-Near-Zero Materials, Phys. Rev. Lett. 97, 157403 (2006).

[48] Y. Wu and J. Li, Total reflection and cloaking by zero index metamaterials loaded with rectangular dielectric defects, Appl. Phys. Lett. 102, 183105 (2013). 